\title{
A New Design of Three-Legged Robot of Mechanical and Control System
}

\author{
Cui Haotian,Yang Wang,Li Qingqing,Yu yueqing,Ma Lizheng,Wang Yumo \\ Beijing University of Technology \\ Beijing, China \\ haotian.cui.work@gmail.com; great03@163.com
}

\begin{abstract}
A new type of three-legged robot design scheme is proposed and the movement control of it is studied in this paper. Spatial crank rocker mechanism (RSSR) is used to will the motor rotation into a leg swinging in mechanical design. Advanced reduced instruction-set computer machines (ARM) chip, wireless communication module and magnetic encoder are used in controller design. Proportional and differential (PD) closed loop control arithmetic is used to realize the control of the robot. The robot realizes the movement of straight walk, curve walk, turning in situ, jumping and so on. It has a broad application prospect.
\end{abstract}

Keywords-three legged robot; motion control; wireless remote control

\section{INTRODUCTION}

Now the bipedal or multiped robots emerge in endlessly. Bipedal robots walk more agilely than multiped robots. However, they lose their balance and fall very easily. So the control arithmetic of bipedal robots is complex. Multiped robots are more stable than bipedal robots. While, redundant legs lead to mutual interference which limits the flexibility of multiped robots.

More and more scientists are attracted by three legged robots with agility and stability. There are few design schemes of three legged robots in and abroad. A kind of three legged robot is developed successfully by scientists in Virginia Tech University [1]. However, the motion of the robot is very slow. Besides, the servo is used to make a kind of three legged robot by some robot lovers [2]. But it is so difficult to make the control arithmetic that the robot walks randomly.

A new type of three legged robot design scheme is proposed and the movement control of it is studied in this paper. It is named Trinibot by us. Solidworks is adopted to finish the mechanical design. RSSR is used to will the motor rotation into a leg swinging. Microcontrollers, wireless communication module and magnetic encoder are used in controller design. The legged is drove independently by the motor. The PD closed loop control arithmetic is used to realize the control of the robot. The movements of straight walk, curve walk, turning in situ, jumping are implemented by the robot in the end.

\section{MECHANICAL SYSTEM}

The whole mechanical system can be divided into driving gear, transmission system and actuator, as shown in Fig. 1. The coreless motor and the model ship battery are selected for driving gear. A set of three-level gear transmission system is used to reduce the output rotate speed and increase the output torque. The module of the gear is 0.5 and the resultant gear ratio is 85.5. The modeling diagram of transmission system is shown in Fig. 2. RSSR is used as actuator to will the motor rotation into a leg swinging in mechanical design, as shown in Fig. 3.

\section{CONTROL SYSTEM ARCHITECHTURE}

The electric control system architecture of the Trinibot is built based on dual ARM Cortex-M3 [3] microcontrollers manufactured by STMicroelectronics. The motion control of

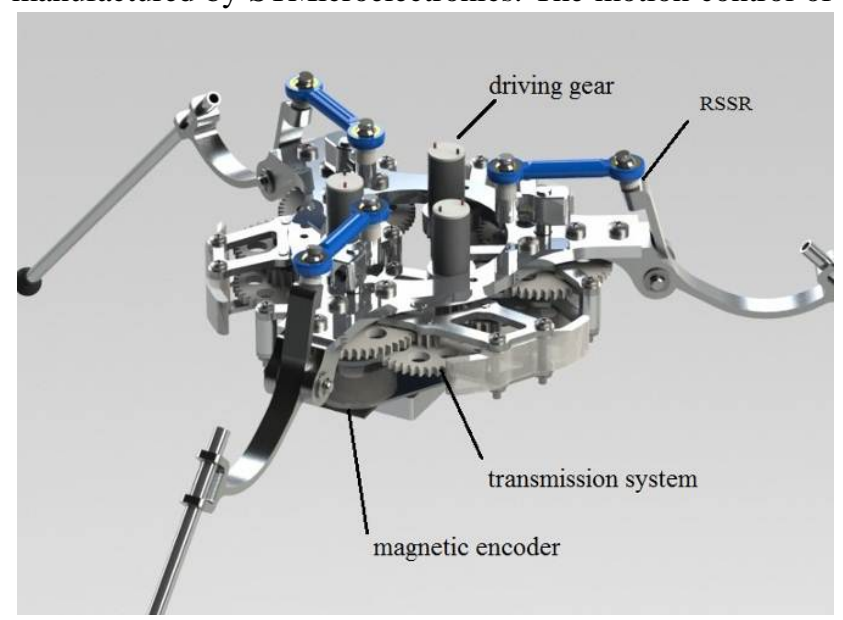

Figure 1. The whole mechanical system of the robot

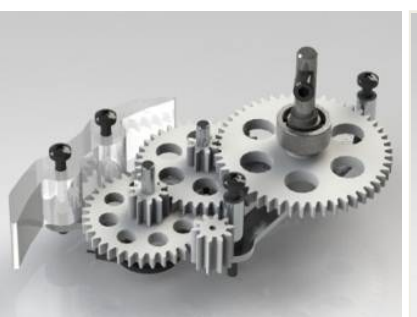

Figure 2. Gear transmission system

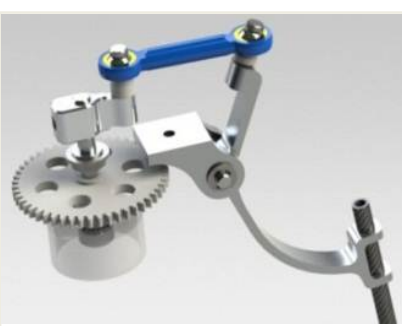

Figure 3. RSSR actuator three brushed DC motors, high level gait planning, wireless communication and tasks scheduling are handled by dual ARM cortex-M3 microcontrollers. In addition, the minimum volume and weight of The Trinibot's DC motor servo driver architecture benefit from the Magnetic Rotary Encoder manufactured by Austriamicrosystems. The integration of all electronic elements in a dual-layer PCB improves the 
compaction, satiability and reducing the weight of the robot. The hardware architecture of the Trinibot control system is showed at Fig. 4.

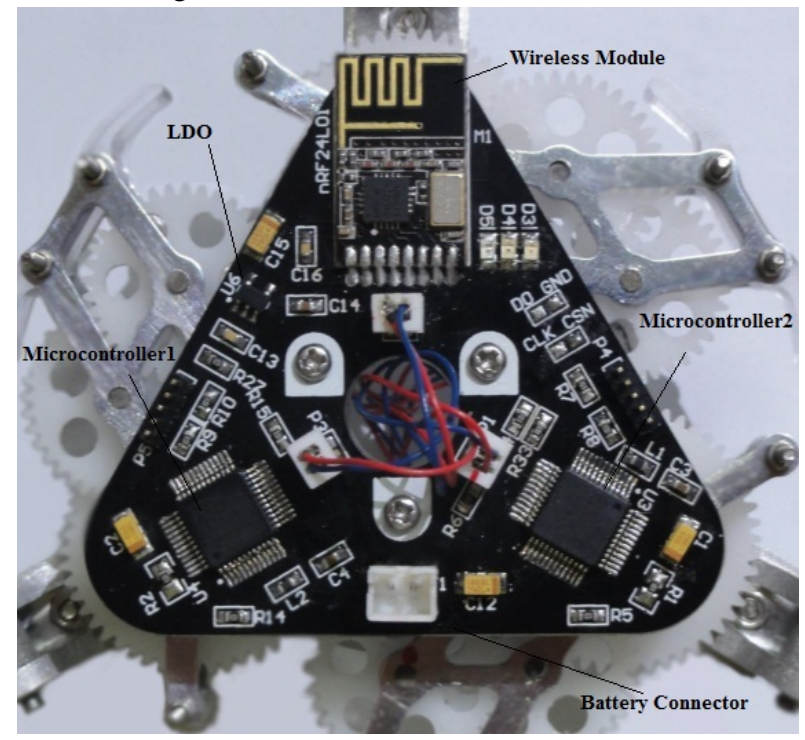

Figure 4. The hardware architechture of the Trinibot control system

\section{A. Motion control}

The motion control of brushed DC motors are composed by Microcontroller 1, monolithic integrated H-bridge driver, DC motors, current-to-voltage converter and Magnetic rotary encoder. First, Microcontroller 1 receives servo motor trajectory data from Microcontroller 2, three absolute position data of DC motor gearbox output axis by Magnetic rotary encoder and current data amplified by Op-amp in real time. Second, Microcontroller 1 calculates the output data using PD control algorithm [4]. And then, the output data are converted to three channel PWM signal. Finally, three Hbridge driver ICs amplifies the PWM signal and control three DC motor spinning. Therefore, the DC motor works as a servo machine. The architecture of control system block diagram is showed at Fig. 5. The absolute rotary encoder AS5040 is noteworthy. AS5040 [5] is a contactless magnetic rotary encoder for accurate angular measurement over a full turn of $360^{\circ}$. It is a system-on-chip, combining integrated Hall elements, analog front end and digital signal processing in a single device. To measure the angle, only a simple twopole magnet, rotating over the center of the chip, is required. The magnet may be placed above or below the IC. The absolute angle measurement provides instant indication of the magnet's angular position with a resolution of $0.35=$ 1024 positions per revolution.

\section{B. The hardware implementing of high-level gaits planning}

Microcontroller 2 receives commands, include forward, turn around, rotation and locomotion velocity, from $2.4 \mathrm{GHz}$ wireless module [6] via SPI (Series Peripheral Interface) bus. Microcontroller 2 deals with the wireless communication tasks, meanwhile, it translates commands from the gaits to motor motion trajectory.

\section{The power supply section}

There are two parts in the robot electronic system, one is power supply, used for DC motor driver, the other is logical supply, used for microcontrollers, wireless communication module, encoder and Op-Amp. We choose a 3.7V 280mAH 20C Li-PO battery for power supply. Logical part supplied by output from a 3.3V LDO (Low Dropout Regulator) CAT6219.

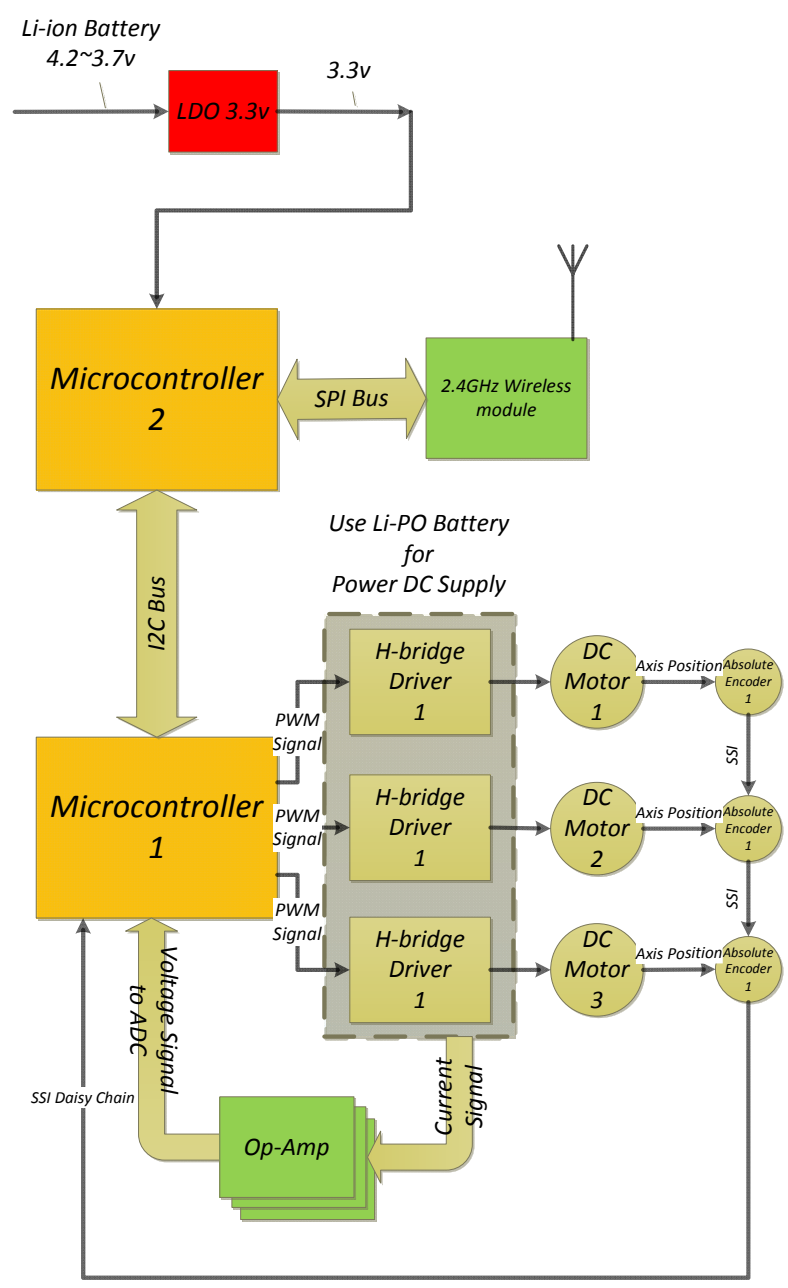

Firure 5. The block diagram of the control system 


\section{BRUSHED DC MOTOR MOTION CONTROL STRATEGIES}

The three-legged of the Trinibot must be subordinated to the trajectory commands transmitted from Microcontroller 2 real time. Therefore, incorporate servo motion control is required. Through the introduction in III, three servo control loop could consists of DC motor, gearbox, encoder, microcontroller and Op-Amp. Control loop block diagram is showed in Fig. 6.

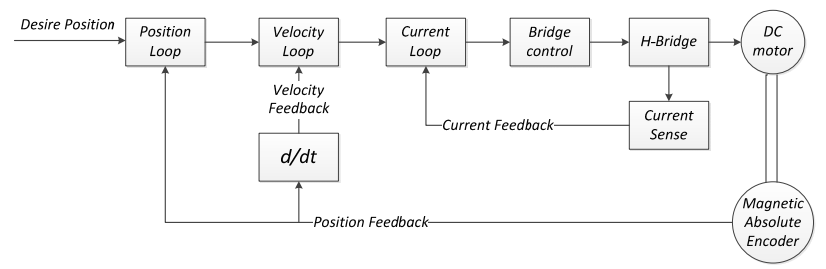

Figure 6. Control loop block diagram

\section{A. Servo control algorithm [7]}

The servo control system includes current loop, velocity loop and position loop. Current loop ensure the motor armature current tracks the current command real time and accurately so that provide sufficient accelerate rotary torque and improve the rapid respond of the DC motor. Velocity loop improves the robustness of anti-disturbance and inhibition of velocity fluctuation. Position loop provides the static precision of system and performance of dynamic tracking.

\section{B. Implementing of feedback control loop algorithm}

The current, velocity and position loop used in servo control loop are built by PD (Proportion Differentiation) control algorithm. The advantage of PD algorithm is better performance in condition where the precise mathematic module of the system is unknown. The feedback control loop diagram as showed in Fig. 7.

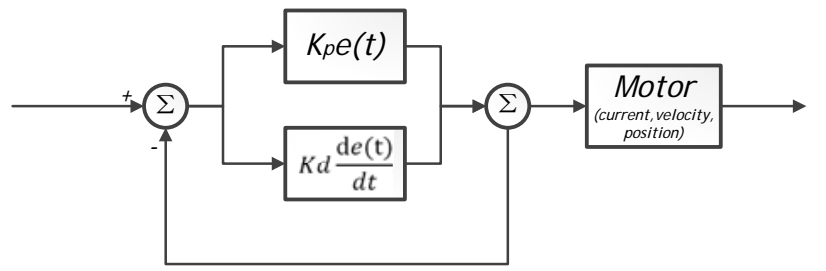

Figure 7. The feedback control loop diagram

The $\mathrm{C}$ code is written below depending on the PD algorithm [8]:

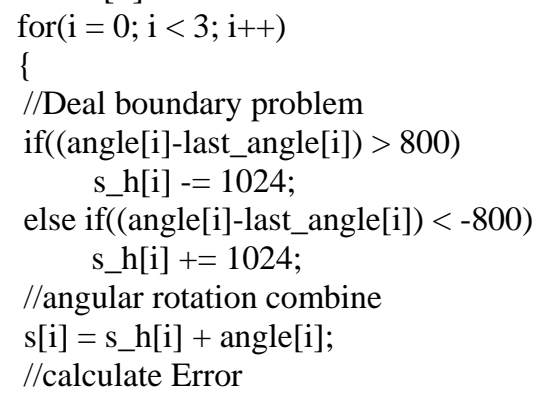

PositionError[i] = target_s[i]-s[i];

dPositionError[i] $=($ target_s[i] $-\mathrm{s}[\mathrm{i}])$ - (last_target_s[i]

- last_s[i]);

//calculate output

$\operatorname{pwm}[\mathrm{i}]=\mathrm{KP} *$ PositionError $[\mathrm{i}]+$

KD*dPositionError[i] ; //PD control

\}

Motor0_set_speed(pwm[0]);

Motor1_set_speed(pwm[1]);

Motor2_set_speed(pwm[2]);

\section{Experiment of servo control system}

In this section, we propose two experiment of the servo control system. First, put the Trinibot on a horizontal surface so that three-legged of the Trinibot carry a force produced by its gravity and three servo motor output axis bears reverse load torque. In this condition, we transmit a step command, allow three servo motors to rotate 180 degrees at the same time, at a time. The robot drops from highest point to the lowest. And then, allow three servo motors to rotate 180 degrees at the same time again, the robot rises from lowest to highest. Fig. 8 shows the performance, tracking the step command, in time domain. Second, we give a ramp signal to three servo motor limit from 0 degree to 360 degree. The performance is showed at Fig. 9.

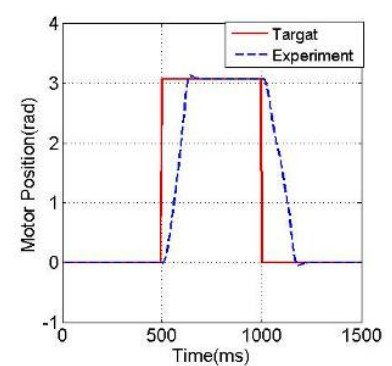

Figure 8. Step response curve

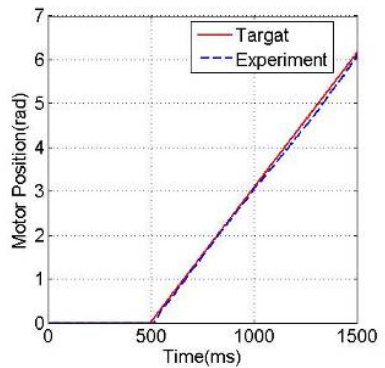

Figure 9. Ramp response curve

\section{THE ACTION FORM AND ANALYSIS}

The three legged robot achieves walking function by moving forward the center of gravity when a leg is raised. When the cranks of the other two legs are at the same phase and a leg is raised, the robot walks straightly. The sketch map is shown in Fig. 10. When the cranks of the other two legs are at the different phase, the robot walks along the curve. The sketch map is shown in Fig. 11. When the phase difference of the robot between crank are 0 degrees and the three legs moving together, the three legged robot achieve the movement of going up and down. The sketch map is shown in Fig. 12. When the phase difference of the robot between crank are 120 degrees and the three legs moving together, the three legged robot achieve the movement of turning in situ. The sketch map is shown in Fig. 13. The analysis of motion is done by MATLAB. The displacement 
and speed curve of the point contacted with the ground is got .The curve is shown in Fig. 14.

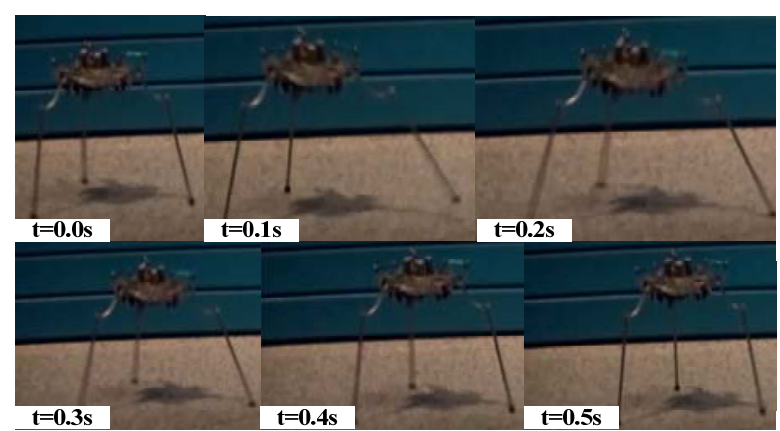

Figure 10. The sketch map of straight walk

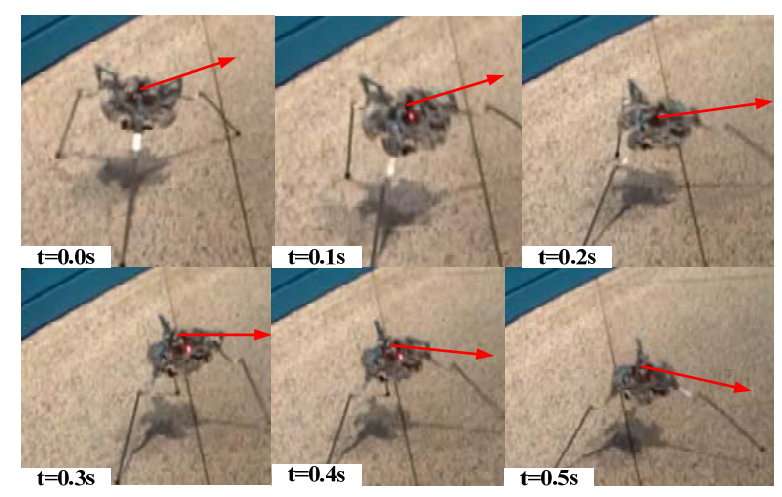

Figure 11. The sketch of curve walk

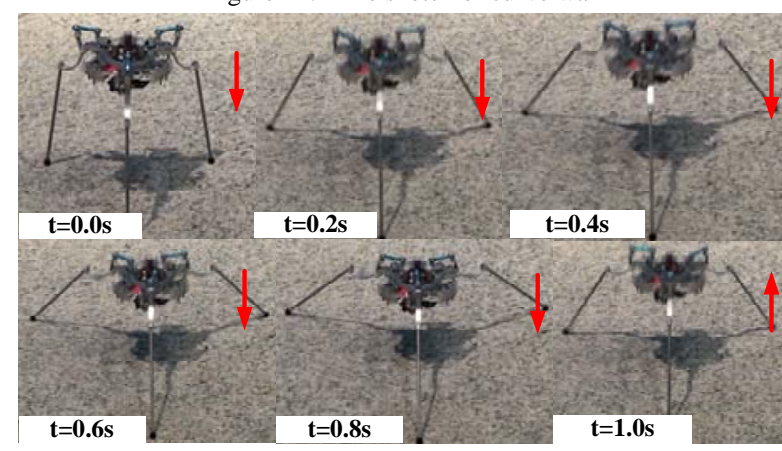

Figure 12. The sketch of promotion and demotion

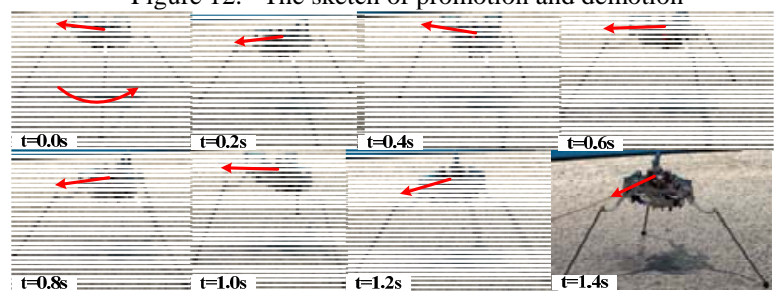

Figure 13. The sketch of turning in suit

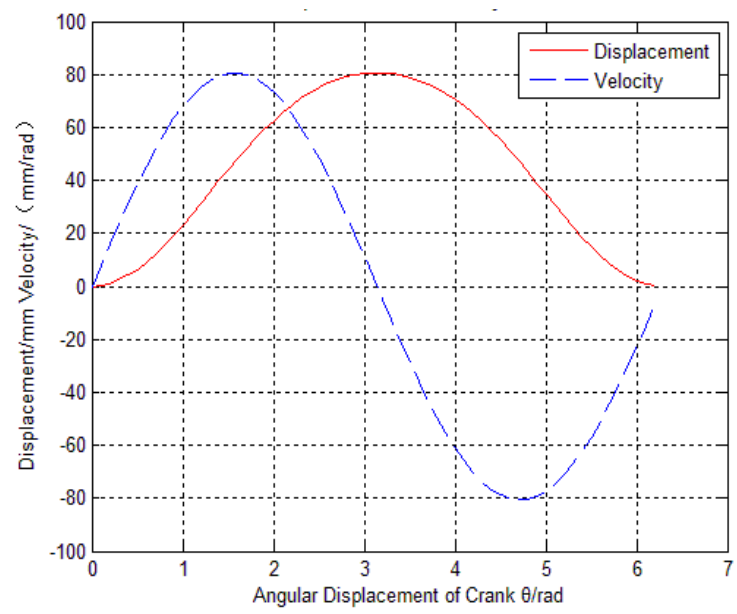

Figure 14. The displacement and speed curve

\section{CONCLUSION}

In this paper, a new type of three legged robot design scheme is proposed and RSSR is used to will the motor rotation into a leg swinging in mechanical design.

The movement control of the three legged robot is studied. The hardware and software is designed independently to realize the movements of straight walk, curve walk, turning in situ, jumping of the robot.

[1] Bokman Lim, Beobkyoon Kim, F.C. Park and D.W.Hong, "Movement Primitives for Three-legged Locomotion over Uneven Terrain”, 2009

[2] Jonathan W. Hurst, Joel E. Chestnutt and Alfred A. RizZj, “An Actuator with Physically Variable Stiffness for Highly Dynamic Legged Locornotion”, 2004

[3] STMicroelectronics group of companies, "STM32F10xxx Reference Manual English”, April, 2010.

[4] Asif Sabanovic, Kouhei Ohnishi, "Motion Control Systems”,2011

[5] Austriamicrosystems, “AS5040_Datasheet_v2_01”, 2011

[6] Sylvain Martel, Mark Sherwood, Chad Helm, William Garcia de Quevedo. Timothy Fofonoff, Robert Dyer, John Bevilacqua, Joshua Kaufman, Omar Roushdy, and Ian Hunter, "THREE-LEGGEWDI RELESS MINIATURE ROBOTS FOR MASS-SCALEO PERATIONS AT THE SUB-ATOMICSC ALE”, 2001

[7] Riazollah Firoozian, "Servo Motors and Industrial Control Theory", 2009.

[8] Youngjin Choi, Wan Kyun Chung, "PID Trajectory Tracking Control for Mechanical Systems”, 2004 